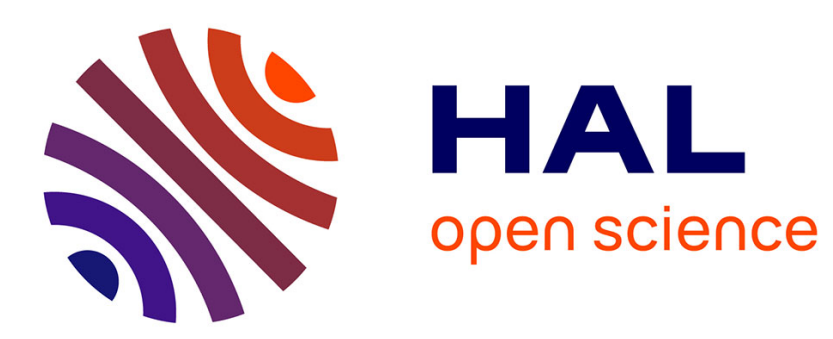

\title{
Efficient Raman converter in the yellow range with high spatial and spectral brightness
}

Minh Chau Phan Huy, Philippe Delaye, Gilles Pauliat, Sylvie Lebrun

\section{To cite this version:}

Minh Chau Phan Huy, Philippe Delaye, Gilles Pauliat, Sylvie Lebrun. Efficient Raman converter in the yellow range with high spatial and spectral brightness. Applied optics, 2018, 57 (24), pp.6892. 10.1364/AO.57.006892 . hal-02326736

\section{HAL Id: hal-02326736 \\ https://hal.science/hal-02326736}

Submitted on 25 Oct 2019

HAL is a multi-disciplinary open access archive for the deposit and dissemination of scientific research documents, whether they are published or not. The documents may come from teaching and research institutions in France or abroad, or from public or private research centers.
L'archive ouverte pluridisciplinaire HAL, est destinée au dépôt et à la diffusion de documents scientifiques de niveau recherche, publiés ou non, émanant des établissements d'enseignement et de recherche français ou étrangers, des laboratoires publics ou privés. 


\title{
Efficient Raman converter in the yellow range with high spatial and spectral brightness
}

\author{
Minh Châu Phan Huy, Philippe Delaye, Gilles Pauliat, Sylvie Lebrun \\ Laboratoire Charles Fabry, Institut d'Optique, CNRS, Univ Paris-Sud, 2 av. A. Fresnel, \\ 91127 Palaiseau cedex, France
}

\begin{abstract}
We present a Raman converter emitting at $583 \mathrm{~nm}$ on the second Stokes order of a line of propan-2-ol pumped by a microlaser at $532 \mathrm{~nm}$ in the sub-nanosecond regime. We used a mixture of liquids to adapt the transmission band of a photonic bandgap fiber. The internal conversion efficiency is $67 \%$ in photon numbers and the output power is $1.06 \mathrm{~mW}$, corresponding to a maximum peak power of $338 \mathrm{~W}$. The beam delivered by the converter presents a Gaussian spatial structure and a high spectral brightness, typically more than 5 times higher than supercontinuum sources in this spectral range.
\end{abstract}

Keywords : Stimulated Raman scattering, hollow core photonic crystal fibers, wavelength converter

\section{Introduction}

Today there is a growing demand for pulsed visible and UV laser sources in biological applications using fluorescence such as bioimaging or DNA sequencing or environmental applications such as atmospheric monitoring. In these areas compact laser systems emitting in the microjoule range one or several "exotic" wavelengths with a good beam quality and a high spectral purity would offer new opportunities for instance in order to excite the increasing number of existing fluorophores [1] or for the realization of new microlidars [2]. Solutions such as supercontinuum sources [3] or OPOs [4] are expensive, cumbersome and oversized when only a few fixed discrete wavelengths are useful. Laser diodes can emit over a large number of wavelengths in the visible range but have many drawbacks such as the poor beam quality and the low peak power in the pulsed regime, limited to a few tens of watts. Moreover to sample the visible spectrum one needs to provide several laser diodes at the expense of extra costs. Microlasers are diode pumped solid-state lasers emitting pulses in the microjoule range with a repetition rate that can vary from $\mathrm{Hz}$ to a few hundred of $\mathrm{kHz}$. These lasers are 
very popular for their compactness and their user-friendly aspect but commercial ones are mostly working at $1064 \mathrm{~nm}$. They can be followed by a non linear stage to emit at $532 \mathrm{~nm}$ and possibly at $355 \mathrm{~nm}$. Extending these achievable wavelengths to "on demand" discrete visible wavelengths would be of great interest for non-laser-specialized researchers. Raman effect has been widely used to generate new wavelengths. Starting from a pump source at a fixed wavelength reaching higher wavelengths is quite easy since the phase-matching condition is automatically satisfied. The Raman medium can be a crystal, a gas or a liquid. With a crystal, the systems are quite complex and not versatile. For example, in [5] in order to generate a given wavelength in the yellow range the authors need to develop a special pump source in the infrared based on Raman effect at first and then insert a nonlinear crystal in the cavity for frequency doubling. Changing the emitted wavelength needs to change several components and a realignment of the whole system. The conversion efficiency remains quite low, typically a few percents. In order to consequently increase the density of pump power over large interaction lengths capillaries or special fibers filled with nonlinear liquids have been studied $[6,7]$. Even if the total conversion efficiency of the pump towards the Stokes lines can be high the energy of the pump is spread over all these lines generated through Raman cascade effect. In order to stop this cascade effect and to optimize the transfer of energy of the pump to a given Stokes order we propose to use hollow-core photonic crystal fibers (HC-PCF) filled with liquids. In such fibers, light can propagate in the core over long distances on small areas with low losses even if the refractive index of the core is smaller than the one of silica [8]. Multiline UV and visible Raman converters in hydrogen-filled HC-PCF have been fabricated [9]. In this reference, many Raman lines and Stokes orders are excited at the same time giving birth to 8 to 23 new wavelengths according to the fiber that is used. The use of liquids with refractive index different from unity offers another degree of freedom. In HC-PCF the photonic bandgap guidance opens spectral transmission bands that can be adapted through the control of the refractive index of the material that fills the holes [10]. In previous works, we demonstrated the efficient conversion of the pump to a single Stokes order in a HC-PCF filled with ethanol by stopping the Raman cascade at the first Stokes order $[11,12,13]$. The pump source emitted at $532 \mathrm{~nm}$ sub-nanosecond $\mu \mathrm{J}$ pulses and the conversion efficiency at high pump power to the first Stokes order of ethanol at $630 \mathrm{~nm}$ was $70 \%$ in photon numbers [12]. In particular we showed that despite the liquid filled fiber was slightly multimode (the used fiber structure is optimized for good transmission properties when empty) an emission on a single quasi Gaussian mode was possible [11]. The real mode shape revealed the hexagonal symmetry of the cladding structure. We also showed that the temporal shape of the pulses can 
be perfectly explained by a quasi-steady state regime modelling of the Raman mechanisms i.e. instantaneous response of the Raman effect at the pulse duration time scale $[12,13]$. We experimentally measured a long-term stability only limited by the pump beam stability [12]. All these characteristics have been observed for different liquids at different Stokes wavelengths [13]. After these proofs of principle we propose to build in the present work an efficient Raman converter emitting in the yellow range at the target wavelength of $583 \mathrm{~nm}$. Indeed this wavelength is interesting for biological applications since it matches an absorption line of several fluorophores, such as Bodipy 581/591 or X-Rhodamine for example [1]. We describe firstly the design of this new converter. The choice of the active Raman liquid and the mixture of liquids to be used to adjust the refractive index and the band position is discussed. Then we present the experimental setup and the results. The internal conversion efficiency from the pump at $532 \mathrm{~nm}$ to the target wavelength at $583 \mathrm{~nm}$ is high $(67 \%$ in photon numbers) despite working on the second Stokes order of the Raman cascade. The new wavelength is emitted in a quasi-Gaussian spatial beam with a high spectral brightness.

\section{Design of the Raman converter emitting at $583 \mathbf{~ n m}$}

The Raman converter is based on the Amplified Spontaneous Emission principle, photons emitted through spontaneous Raman scattering being further amplified by stimulated Raman scattering. It is thus very simply implemented as requiring just a pump operating in a single pass scheme in the nonlinear medium. Liquids as any material present complex Raman spectra with many lines among which the one with the highest Raman gain coefficient $g_{R} 1$ is called the main Raman line. Other lines with lower Raman gain coefficients $g_{R i}$ are called secondary Raman lines. For a given line, the Raman threshold can be defined when the parameter $\gamma=g_{R} \frac{P_{p} \times L}{A_{e f f}}$ is approximately equal to 25 [14]. $\mathrm{g}_{\mathrm{R}}$ is the Raman gain coefficient, $\mathrm{P}_{\mathrm{p}}$ is the internal peak pump power, $\mathrm{A}_{\mathrm{eff}}$ the effective area and $\mathrm{L}$ the interaction length. When increasing $\mathrm{P}_{\mathrm{p}}$ and assuming no losses, before its saturation the Stokes power varies as $\exp (\gamma)$. So, around threshold, if $\frac{g_{R i}}{g_{R 1}} \precsim 0.7$, the Stokes power of the main line will be more than 1800 times higher than the Stokes power of the secondary Raman line. So we propose this value of 0.7 as a good criterion to say that, as we are working in the steady-state regime, Stimulated Raman Scattering (SRS) occurs on the main Raman line only, secondary lines emission being neglected. Above threshold the pump begins to be depleted by energy transfer toward the main line and no more power is available to amplify the secondary lines, increasing again the 
effect. When $\frac{g_{R i}}{g_{R 1}}$ becomes close to unity, it is difficult to identify the main Raman line from a secondary line. In this case competition occurs between the two lines and a solution to favor one line is to put extra-losses on the other one. Indeed the amplification gain of the unwanted line varies as $\exp (\gamma-\alpha L)$ where $\alpha$ represents the linear losses and hence vanishes when $\gamma<$ $\alpha L$.

In this work we want to shift the pump wavelength at $532 \mathrm{~nm}$ to the target wavelength of $583 \mathrm{~nm}$ by using SRS in a liquid. Such a wavelength shift corresponds to a Raman shift around $1640 \mathrm{~cm}^{-1}$ which is not directly achievable with common and not harmful Raman liquids we want to use $[15,16]$. It also corresponds to the second Stokes order of a line with a Raman shift around $820 \mathrm{~cm}^{-1}$. This shift matches with a Raman line of alcohols. To choose the most convenient alcohol, we have to calculate the Raman gain coefficients. For that, we have used the formula demonstrated in [17] and considered the depolarization ratio $\rho_{S}$ which adds a correction factor $\frac{1}{1+\rho_{S}}$ to it [18]. This formula becomes :

$$
g_{R}=\frac{1}{1+\rho_{S}} \frac{8 \pi}{\Delta v_{F W H H}} \frac{N c^{2}}{\omega_{S}^{3} \hbar n_{S}^{2}}\left(\frac{\partial \sigma}{\partial \Omega}\right)
$$

For the liquids and lines we use in this work, $\rho_{S}$ is much smaller than one and brings only a minor correction, but has to be considered in some other liquids or lines. $\Delta v_{F W H H}$ is the full width at half height of the spontaneous Raman line, $\mathrm{N}$ is the molecular density, $\mathrm{c}$ is the velocity of light, $\omega_{\mathrm{s}}$ and $\mathrm{n}_{\mathrm{s}}$ are the Stokes frequency and refractive index. $\frac{\partial \sigma}{\partial \Omega}$ is the total differential scattering cross-section [19]. In [20] this cross-section is given for a pump wavelength of $488 \mathrm{~nm}$. As this parameter is wavelength dependent, we have to calculate it for the wavelengths we are interested in according to the following formula [21] :

$$
\frac{\partial \sigma}{\partial \Omega}=\frac{A \omega_{S}^{4}}{\left(\omega_{i}^{2}-\omega_{p}^{2}\right)^{2}}
$$

$\mathrm{A}$ is a constant of proportionality, $\omega_{\mathrm{p}}$ is the pump frequency, $\omega_{\mathrm{s}}$ is the Stokes frequency and $\omega_{\mathrm{i}}$ a UV material resonance frequency. In the liquids that we study, $\omega_{p} \ll \omega_{i}$, so we can consider that $\frac{\partial \sigma}{\partial \Omega}$ is proportional to the fourth power of $\omega_{\mathrm{s}}$.

In alcohols there are usually two strong Raman bands, one around 2800-3000 $\mathrm{cm}^{-1}$ (denoted band I in the following) and one around $800-1040 \mathrm{~cm}^{-1}$ (denoted band II). Each band contains 
several Raman lines. In tables 1 and 2, we have computed the Raman gain coefficients $g_{R}$ in several alcohols for the strongest Raman line in band I (denoted $g_{R, I}$ ) and for the strongest Raman line in band II (denoted $\mathrm{g}_{\mathrm{R}, \mathrm{II}}$ ) from the figures published in $[20,22]$ at the pump wavelength $\lambda_{p}$ of $532 \mathrm{~nm}$. We calculated $g_{R, I I}$ for the first Stokes order emitting at $\lambda_{1 \mathrm{~s}}\left(\mathrm{~g}_{\mathrm{R}, \mathrm{II}}\right.$ at $\left.\lambda_{\mathrm{p}}\right)$ and for the second Stokes order emitting at $\lambda_{2 \mathrm{~s}}\left(\mathrm{~g}_{\mathrm{R}, \mathrm{II}}\right.$ at $\lambda_{1 \mathrm{~s}}, \lambda_{1 \mathrm{~s}}$ being the pumping wavelength) and finally the ratio $\frac{g_{R, I I} \text { at } \lambda_{1 s}}{g_{R, I} \text { at } \lambda_{p}}$.

In methanol and ethanol, the ratio $\frac{g_{R, I I} \text { at } \lambda_{1 s}}{g_{R, I} \text { at } \lambda_{p}}$ is largely below 0.7 , so we expect that SRS will occur on the Raman line of band I only. Propanol has two isomers, propan-1-ol and propan-2ol. In the literature we did not find values of Raman cross sections in propan-1-ol, so we are not able to calculate the Raman gain. However, in [23] and [24] the authors both evaluated that the intensity of the Raman line at $2876 \mathrm{~cm}^{-1}$ is higher than the intensity of the Raman line at $860 \mathrm{~cm}^{-1}$. This assumption is also stressed by a visual comparison of the Raman spectrum of propan-1-ol with the Raman spectrum of ethanol, showing the same global trends [16]. This makes us believe that the strongest line in band I is the main Raman line in propan-1-ol, as for methanol and ethanol, and will be strongly favored at the expense of band II.

For propan-2-ol, more data are available in the literature and enable to calculate the Raman gains $[20,22]$. As can be seen in table 1 the Raman line of propan-2-ol at $2882 \mathrm{~cm}^{-1}$ has a gain of $2.43 \times 10^{12} \mathrm{~W}^{-1} \mathrm{~m}$. As can be seen in table 2 the Raman line at $819 \mathrm{~cm}^{-1}$ has a gain of $2.50 \times 10^{12} \mathrm{~W}^{-1} \mathrm{~m}$ for the first Stokes order and a gain of $2.37 \times 10^{12} \mathrm{~W}^{-1} \mathrm{~m}$ for the second Stokes order we want to generate.

\begin{tabular}{|c|c|c|c|}
\hline Liquid & Raman shift $\left(\mathrm{cm}^{-1}\right)$ & $\lambda_{1 \mathrm{~s}}(\mathrm{~nm})$ & $\mathrm{g}_{\mathrm{R}, \mathrm{I}}$ at $\lambda_{\mathrm{p}}$ \\
\hline Methanol & 2837 & 626 & 2.16 \\
\hline Ethanol & 2928 & 630 & 2.92 \\
\hline Propan-2-ol & 2882 & 628 & 2.43 \\
\hline
\end{tabular}

Table 1. Estimated Raman gains (in $\times 10^{12} \mathrm{~W}^{-1} \mathrm{~m}$ ) of different alcohols for the strongest Raman line in band $\mathrm{I}$. The pump wavelength is $\lambda_{p}=532 \mathrm{~nm}$. $\lambda_{1 \mathrm{~s}}$ is the wavelength of the first Stokes order.

\begin{tabular}{|c|c|c|c|c|c|c|}
\hline Liquid & Raman shift $\left(\mathrm{cm}^{-1}\right)$ & $\lambda_{1 \mathrm{~s}}(\mathrm{~nm})$ & $\lambda_{2 \mathrm{~s}}(\mathrm{~nm})$ & $\mathrm{g}_{\mathrm{R}, \mathrm{II}}$ at $\lambda_{\mathrm{p}}$ & $\mathrm{g}_{\mathrm{R}, \mathrm{II}}$ at $\lambda_{1 \mathrm{~s}}$ & $\frac{\mathrm{g}_{\mathrm{R}, \mathrm{II}} \text { at } \lambda_{1 \mathrm{~s}}}{\mathrm{~g}_{\mathrm{R}, \mathrm{I}} \text { at } \lambda_{\mathrm{p}}}$ \\
\hline Methanol & 1037 & 563 & 598 & 0.57 & 0.54 & 0.25 \\
\hline Ethanol & 881 & 558 & 587 & 1.04 & 0.99 & 0.34 \\
\hline Propan-2-ol & 819 & 556 & 583 & 2.50 & 2.37 & 0.97 \\
\hline
\end{tabular}


Table 2. Estimated Raman gains (in $\times 10^{12} \mathrm{~W}^{-1} \mathrm{~m}$ ) of different alcohols for the strongest Raman line in band II. The pump wavelength is $\lambda_{p}=532 \mathrm{~nm} . \lambda_{1 \mathrm{~s}}\left(\lambda_{2 \mathrm{~s}}\right)$ is the wavelength of the first (second) Stokes order.

Contrary to the other alcohols studied, in propan-2-ol, $\frac{g_{R, I I} \text { at } \lambda_{1 s}}{g_{R, I} \text { at } \lambda_{p}}$ is very close to unity. So in comparison with ethanol, methanol and propan-1-ol this liquid is the most favorable for SRS emission on the $819 \mathrm{~cm}^{-1}$ line. As $\mathrm{g}_{\mathrm{R}, \mathrm{II}}$ in propan-2-ol is almost the same as $g_{R, I}$ in ethanol for which we already demonstrated SRS in ethanol in a Raman converter on the $2928 \mathrm{~cm}^{-1}$ line [11,12], we are confident that it will be high enough to generate SRS in this new converter. So we decided to use propan-2-ol to generate SRS on the second Stokes order of the line at $819 \mathrm{~cm}^{-1}$. Our objective is then twofold : 1) to stop the Raman cascade at the second Stokes order of the line at $819 \mathrm{~cm}^{-1}$ in order to optimize the pump transfer energy on the target wavelength and 2) to prevent SRS on the line at $2882 \mathrm{~cm}^{-1}$ by adding extra-losses on it.

We have used a $1 \mathrm{~m}$ long HC-PCF with a transmission band centered around $1 \mu \mathrm{m}$ (ref. NKT Photonics, HC 1060-02). In order to shift the transmission band around $580 \mathrm{~nm}$, the fiber has to be filled with a liquid having a refractive index around 1.39 [10,13]. As the refractive index of propan-2-ol is 1.37 , one needs to mix it with a high index liquid. We have chosen to use DMSO (Dimethyl Sulfoxyde) as the diluent, as it mixes well with alcohols and as its refractive index being 1.48, allowing to keep a high proportion of the alcohol in the mixture. The proportions of the liquids were $11 \%$ of DMSO and $89 \%$ of propan-2-ol in volume. This weak dilution ensures that the Raman gain of DMSO in the mixture remains much smaller than the Raman gain of propan-2-ol in the mixture, so that the converter will not emit on a Raman line of DMSO. The refractive index of the mixture is firstly measured with an Abbe refractometer at $589 \mathrm{~nm}$. Then a small piece of fiber $(5 \mathrm{~cm})$ is filled with the mixture to measure the transmission band by injecting the light of a supercontinuum laser in it [3]. At the output of this small piece of fiber, we collect and filter the light that comes from the core before sending it to the optical spectrum analyzer (OSA). Some adjustments of the liquid index are sometimes necessary and once the transmission band is satisfactory we fill the fiber of the converter with the liquid thanks to a homemade microfluidic system. Both ends are hermetically closed with micro-tanks [25]. The whole device "liquid HC-PCF + tanks" is called the "Raman converter" (see insert of fig. 2). The transmission bandwidth of the converter may be slightly narrower than the one of the small piece of fiber because of the filtering of the modes along the length of propagation (typically $1 \mathrm{~m}$ ). Another fine adjustment of the liquid index may be necessary. In fig. 1, we show three transmission bands 
of the same fiber, being filled with a liquid mixture having successively a refractive index of 1.384, 1.388 and 1.394. Between two fillings our microfluidic system enables to rinse the fiber to ensure the mixture of liquids is the expected one. The transmission bands were registered by optimizing the level of the output signal of the converter at $532 \mathrm{~nm}$. We can estimate from fig. 1 that the right band edge of the transmission band shifts with a rate of roughly $5 \mathrm{~nm}$ for a change in the refractive index of $10^{-3}$ in the range of indices we use. This estimation is helpful to optimize the position of the transmission band with the refractive index of the liquid mixture. The transmission band is optimized to contain the pump line at $532 \mathrm{~nm}$, the first Stokes order line at $556 \mathrm{~nm}$ and the second Stokes order line at $583 \mathrm{~nm}$. The third Stokes order at $612 \mathrm{~nm}$ and the first Stokes order at $628 \mathrm{~nm}$ of the line at $2882 \mathrm{~cm}^{-1}$ have to be out of the transmission band, so that these lines will not be generated efficiently. From fig. 1 , we can see that converter with $\mathrm{n}=1.394$ is not optimized since the line at $583 \mathrm{~nm}$ suffers from too much losses. We have tested the two other converters $(n=1.384$ and $n=1.388)$ and will present the best results obtained with the converter having $n=1.388$.

\section{Experimental set-up}

The experimental set-up is depicted in figure 2. We have used a frequency doubled microlaser emitting at $532 \mathrm{~nm}$ as the pump source, with a pulse duration of $560 \mathrm{ps}$ (FWHM). The frequency rate repetition is $5.6 \mathrm{kHz}$ and the maximum mean pump power incident on the converter is $5.75 \mathrm{~mW}$ (corresponding to a peak power of $1.8 \mathrm{~kW}$ and an energy per pulse of $1 \mu \mathrm{J})$. The pump beam is focused inside the Raman converter through a microscope objective (x6.3, NA 0.2). At the output of the Raman converter, we collect the light through a second microscope objective (x6.3, NA 0.2). The light is sent in an OSA through a multimode fiber. We measured the power of the different lines emitted by the Raman converter by sending the output beam through a multimode fiber in an OSA used with a low resolution of $10 \mathrm{~nm}$, much larger than the Raman linewidths. The power calibration of the system "multimode fiber + OSA" is firstly made by measuring the power in $\mathrm{mW}$ of the different wavelengths taken separately thanks to coloured filters, put just before the multimode fiber. Knowing the transmission of the coloured filters, we can deduce the power in $\mathrm{mW}$ of the different wavelengths at the output of the converter. Then for each wavelength a mean power in arbitrary unit is calculated from the OSA and can be converted into the power in $\mathrm{mW}$ at the 
output of the converter. Within this method we can have simultaneous measurements of the powers of each wavelength delivered by the Raman converter.

\section{Results}

In fig. 3 we show an example of the transmitted powers of the different lines delivered by the converter versus the incident pump power $P_{\text {in }}$ at $532 \mathrm{~nm}$. The injection of the pump beam in the converter was firstly optimized for low pump powers, before the threshold of the first Stokes order at $556 \mathrm{~nm}$. The pump injection efficiency is then estimated to be $30 \%$. Then we increased $P_{\text {in }}$ until the appearance of the first Stokes order at $556 \mathrm{~nm}$ for $P_{\text {in }}=1.5 \mathrm{~mW}$. Moving slightly the injection microscope enables to optimize the output powers but sometimes led to irreversible damage of the converter (probable burning of impurities or dusts at the input of the converter) so we chose not to optimize the output power at $556 \mathrm{~nm}$ as we were not interested in. This explains why the power at $556 \mathrm{~nm}$ does not grow higher. By continuing to increase $\mathrm{P}_{\text {in }}$ we observed the appearance of the second Stokes order at $583 \mathrm{~nm}$ for $\mathrm{P}_{\text {in }}=3 \mathrm{~mW}$. The first Stokes order saturated, and the second Stokes order then grew without any saturation. Just before the maximum incident pump power we carefully optimized the output power at $583 \mathrm{~nm}$ and obtained a power of $1.06 \mathrm{~mW}$ for the maximum pump power of $5.75 \mathrm{~mW}$. It corresponds to a maximum peak power of $338 \mathrm{~W}$ at $583 \mathrm{~nm}$ and an energy of $0.19 \mu \mathrm{J}$ per pulse. The internal conversion efficiency from the internal pump power to the second Stokes order at $583 \mathrm{~nm}$ is $61 \%$ (or $67 \%$ in photon numbers). For high pump powers, we observed that the pump and the first Stokes order at $556 \mathrm{~nm}$ are not completely depleted which has been previously explained by the Gaussian temporal shape of the pump pulse [12, 13]. At maximum pump power we observed the onset of the third Stokes order at $612 \mathrm{~nm}$ in a core mode and the onset of the first Stokes order of the line in band I at $628 \mathrm{~nm}$ in a cladding mode but the powers were very weak (about a few $\mu \mathrm{W}$ ). This illustrates the difficulty to adjust the transmission band that may still be improved but also shows the efficiency of the SRS in the converter.

The highest achievable resolution of the OSA we used in the present experiment was $50 \mathrm{pm}$ which is in the range of the Raman spectral linewidths of liquids. So even if it was not possible to precisely measure the linewidths of the emitted lines we had an upper limit of these values. Insert in fig. 3 shows an example of the spectrum of the line at $583 \mathrm{~nm}$ obtained at the highest resolution of the OSA. To get a more precise value we used the fact that the 
spectral width of the Stokes output $\Delta \omega_{1 / 2}(\mathrm{FWHH})$ narrows with the gain according to the following formula [26] :

$$
\Delta \omega_{1 / 2} \cong\left(\frac{\ln 2}{2 \gamma \times L}\right)^{1 / 2} \Gamma
$$

$\mathrm{L}$ is the length of the converter, $\Gamma$ is the spontaneous spectral linewidth $(\mathrm{FWHH})$. We took $\mathrm{L}=1 \mathrm{~m}$ and $\Gamma=6.6 \quad \mathrm{~cm}^{-1} \quad[22] . \quad$ To calculate $\gamma$ we took $\mathrm{P}_{\mathrm{p}}=59 \mathrm{~W}$ (see fig. 3). We approximate the Raman gain of propan-2-ol in the mixture by simply multiplying its value obtained in table 2 for its pure form by its proportion in the mixture, i.e. $89 \%$. We obtained $\mathrm{g}_{\mathrm{R}}=2.11 \times 10^{-12} \mathrm{~W}^{-1} \mathrm{~m}$. For $\mathrm{A}_{\text {eff }}$ we took the value measured in a previous work in a Raman converter built with the same HC-PCF and filled with ethanol [11], i.e. $12 \times 10^{-12} \mathrm{~m}^{2}$. We found then $\Delta \omega_{1 / 2}=1.2 \mathrm{~cm}^{-1}$ (corresponding to $47 \mathrm{pm}$ ). The estimated spectral power density at $583 \mathrm{~nm}$ is $22 \mathrm{~mW} / \mathrm{nm}$, which is well above the one obtained in this spectral range by sub-ns supercontinuum sources, typically $700 \mu \mathrm{W} / \mathrm{nm}$ in the $\mathrm{MHz}$ range (among the references of [3] a spectral power density of $4 \mathrm{~mW} / \mathrm{nm}$ has been obtained but in the ps regime).

The output beam of the converter was sent on a diffraction grating after which we observe the spatial patterns of the modes projected on a screen (Fig. 4 (a)). On this saturated image we can see the typical shape of the modes of HC-PCF fibers with an hexagonal symmetry due to the structure of the cladding. The periodic structure of the holes in the cladding is also responsible for the presence of petal lobes around the core. Theses petal lobes can be seen as part of the fundamental mode and carry only a very small percentage of the energy. On the far-field patterns of Fig. 4(b) and (c) we can see that the Stokes lines at $556 \mathrm{~nm}$ and $583 \mathrm{~nm}$ are emitted in quasi-Gaussian modes.

From simulations of the Raman cascade [12] and from previous experimental studies [27] we can estimate that the temporal pulse duration at $583 \mathrm{~nm}$ is shorter than the pump pulse duration (i.e. 560 ps FWHM), an order of magnitude of this pulse duration at maximum pump power is expected to be around 100-200 ps FWHM by the numerical simulations.

In this work we have shown that the exploitation of the various Raman lines of common liquids and the adjustment of the refractive index of the liquid mixtures to finely control the position of the transmission bands broaden the possibilities of our Raman converters. A simple microlaser at $532 \mathrm{~nm}$ associated with different Raman converters will enable to access 
"on demand" discrete wavelengths with high conversion efficiencies in beams with high spectral brightness and good spatial quality, opening new opportunities in biological applications for instance.

\section{Conclusion}

We have designed, built and characterized a Raman converter emitting at $583 \mathrm{~nm}$ on the second Stokes order of a line of propan-2-ol pumped by a microlaser at $532 \mathrm{~nm}$ in the subnanosecond regime. The internal conversion efficiency is $67 \%$ in photon numbers and the output power is $1.06 \mathrm{~mW}$, corresponding to a maximum peak power of $338 \mathrm{~W}$. The beam delivered by the converter presents a Gaussian spatial structure and a spectral brightness more than 5 times higher than supercontinuum sources in this spectral range. The principle of this Raman converter can be adapted to other Raman liquids to deliver other wavelengths in the visible range. These Raman converters have applications in areas such as fluorophore excitation where pulsed low energies at specific discrete wavelengths are needed. Moreover the liquid mixtures method offers a new degree of freedom for the study of other nonlinear effects. For example it may enable to adjust the position of the zero-dispersion wavelength to realize a phase-matching condition in a four-wave mixing process for the generation of correlated photon pairs [28].

\section{Acknowledgements}

The authors thank the "Fondation Coopération Scientifique Campus Paris Saclay" for supporting the work of Minh Châu Phan Huy.

\section{References}

[1] see for example : http://www.thermofisher.com/fr/fr/home/life-science/cellanalysis/labeling-chemistry/fluorescence-spectraviewer.html www.biotech.iastate.edu/facilities/flow/Fluorochromes.pdf

[2] J. Spinhirn, "Micro pulse lidars", IEEE transactions on geoscience and remote sensing, vol. 31 (1), jan. 1993.

[3] https://www.leukos-systems.com/our-products/supercontinuum-lasers/scientific/new-wave https://www.nktphotonics.com/lasers-fibers/product/superk-extreme-supercontinuum-lasers/ 
http://www.yslphotonics.com/Home/Index/Product/index/id/24.html

[4] http://www.elforlight.com/_products/opo_03.htm

[5] T. Omatsu, A. Lee, H. M. Pask, J. Piper, "Passively Q-switched yellow laser formed by a self-Raman composite Nd:YVO4/YVO4 crystal”, Appl Phys B, vol. 97, pp 799-804, 2009.

[6] G. Fanjoux, A. Sudirman, J. C. Beugnot, L. Furfaro, W. Margulis, T. Sylvestre, "Stimulated Raman-Kerr scattering in an integrated nonlinear optofluidic fiber arrangement", Opt Lett., vol 39 (18), pp 5407-10, 2014.

[7] S. Yiou, P. Delaye, A. Rouvie, J. Chinaud, R. Frey, G. Roosen, P. Viale, S. Février, P. Roy, J.-L. Auguste, J.-M. Blondy, "Stimulated Raman scattering in an ethanol core microstructured optical fiber", Opt. Express, 13, 4786 (2005).

[8] R.F. Cregan , B.J. Mangan , J.C. Knight, T.A. Birks , P.S. Russell , P.J. Roberts , D.C. Allan, "Single-Mode Photonic Band Gap Guidance of Light in Air", Science. 1999 Sep 3;285(5433):1537-1539.

[9] Y. Y. Wang, F. Couny, P. S. Light, B. J. Mangan, F. Benabid, "Compact and portable multiline UV and visible Raman lasers in hydrogen-filled HC-PCF", Opt Lett. April 15, 2010, vol. $35, \mathrm{n}^{\circ} 8$, p. 1127-1129.

[10] G. Antonopoulos, F. Benabid, and T. A. Birks, "Experimental demonstration of the frequency shift of bandgaps in photonic crystal fibers due to refractive index scaling," Opt. Express 14,3000-3006 (2006).

[11] S. Lebrun, P. Delaye, R. Frey, and G. Roosen, "High-efficiency single-mode Raman generation in a liquid-filled photonic bandgap fiber", Opt. Lett. vol 32, 5 (2007).

[12] S. Lebrun, C. Buy, P. Delaye, R. Frey, G. Pauliat and G. Roosen, "Optical characterizations of a Raman generator based on a hollow core photonic crystal fibre filled with a liquid", Journal of Nonlinear Optical Physics \& Materials, 19,(1), pp. 101-109 (2010).

[13] S. Lebrun, M. C. Phan Huy, P. Delaye, and G. Pauliat, "Efficient stimulated Raman scattering in hybrid liquid-silica fibers for wavelength conversion", In SPIE/COS Photonics Asia, Optical Design and Testing VII, volume 10021, page 1002104, Beijing, China, October 2016.

[14] M. C. Phan Huy, P. Delaye, G. Pauliat, N. Dubreuil, F. Gérôme, B. Debord, F. Benabid, and S. Lebrun, "Lowering backward Raman and Brillouin scattering in waveguide Raman wavelength converters", Journal of the European Optical Society : Rapid publications, 13:31 (2017).

[15] M. D. Martin and E. L. Thomas, "Infrared difference frequency generation", IEEE Journal of Quantum Electronics, vol. QE-2, n², Aug. 1966. 
[16] Spectral Database for Organic Compounds SDBS : http://sdbs.db.aist.go.jp/sdbs/cgibin/direct_frame_top.cgi

[17] M. Maier, W. Kaiser, J. A. Giordmaine, "Backward Stimulated Raman scattering", Phys. Rev. 177, 580-599 (1969).

[18] S. Porto, “Angular dependence and Depolarization Ratio of the Raman effect", JOSA, 56, 1585-1589 (1966).

[19] L. Shan, G. Pauliat, G. Vienne, L. Tong, and S. Lebrun, "Design of nanofibres for efficient stimulated Raman scattering in the evanescent field", Journal of the European Optical Society : Rapid publications, 8:13030, April 2013.

[20] J. E. Griffiths, "Raman scattering cross sections in strongly interacting liquid systems : $\mathrm{CH} 3 \mathrm{OH}, \mathrm{C} 2 \mathrm{H} 5 \mathrm{OH}$, i-C3H7OH, $(\mathrm{CH} 3) 2 \mathrm{CO}, \mathrm{H} 2 \mathrm{O}$, and D2O", The Journal of Chemical Physics, Vol. 60, nº, 15 March 1974.

[21] W. K. Bischel and G. Black, “Wavelength dependence of Raman scattering cross sections from 200-600 nm", in Excimer Lasers-1983, C. K. Rhodes, H. Egger, and H. Pummer, eds. (American Institute of Physics, New York, 1983), pp. 181-187.

[22] M. J. Colles and J. E. Griffiths, "Relative and absolute Raman scattering cross sections in liquids", The Journal of Chemical Physics, Vol. 56, nº 1 april 1972.

[23] K. Krishnan, “The Raman spectra of organic compounds, Part. I Methyl, Ethyl, n-Propyl and n-Butyl Alcohols", Proceedings of the Indian Academy of Sciences - Section A, Volume 53, Issue 3, pp 151-167, March 1961.

[24] R. W. Wood and G. Collins, “ Raman spectra of a Series of Normal Alcohols and Other Compounds”, Phys. Rev., Volume 42, pp 386-392, November 1932.

[25] S. Lebrun, M. C. Phan Huy, P. Delaye, and G. Pauliat, "Optical device having a liquidcore optical fibre and method for producing such a device", Patent FR 12 58870/ WO 2014/044829 A1.

[26] Chen-Show Wang, "Theory of stimulated Raman scattering”, Phys. Rev. Vol. 182, 2, 1969.

[27] L. Shan, G. Pauliat, G. Vienne, L. Tong, and S. Lebrun, "Stimulated Raman scattering in the evanescent field of liquid immersed tapered nanofibers", Applied Physics Letters, 102:201110, May 2013.

[28] Margaux Barbier, Isabelle Zaquine, Philippe Delaye, “ Spontaneous four-wave mixing in liquid-core fibers : towards fibered Raman-free correlated photon sources ", New Journal of Physics, 17, pp. 053001, May 2015. 


\section{Figure captions}

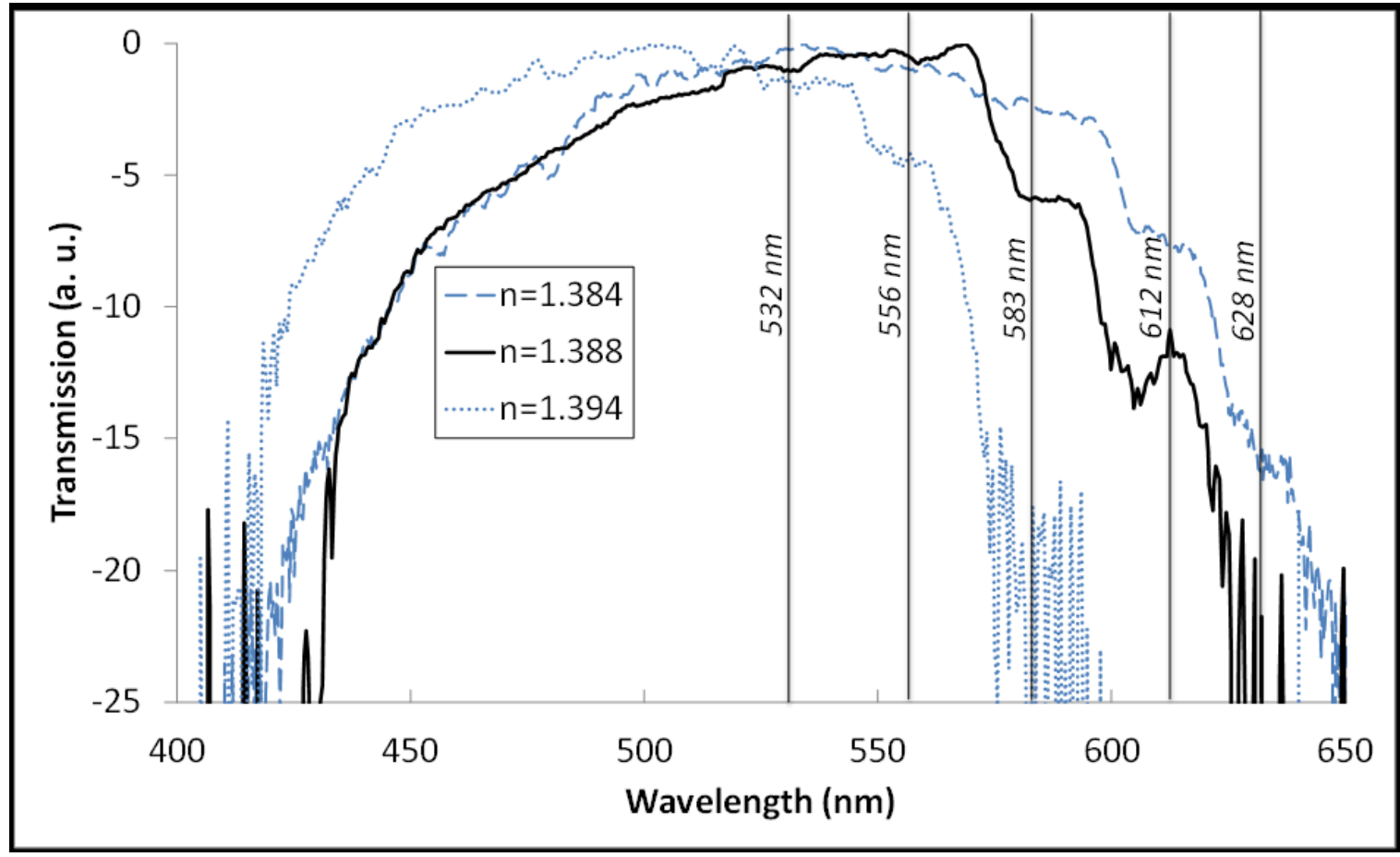

Figure 1. Optimization of the transmission band of a $1 \mathrm{~m}$ long HCPCF 1060-02 filled with a mixture of propan-2-ol and DMSO. The refractive index of the liquid mixture is respectively 1.384 (dashed line), 1.388 (full line) and 1.394 (dotted line).

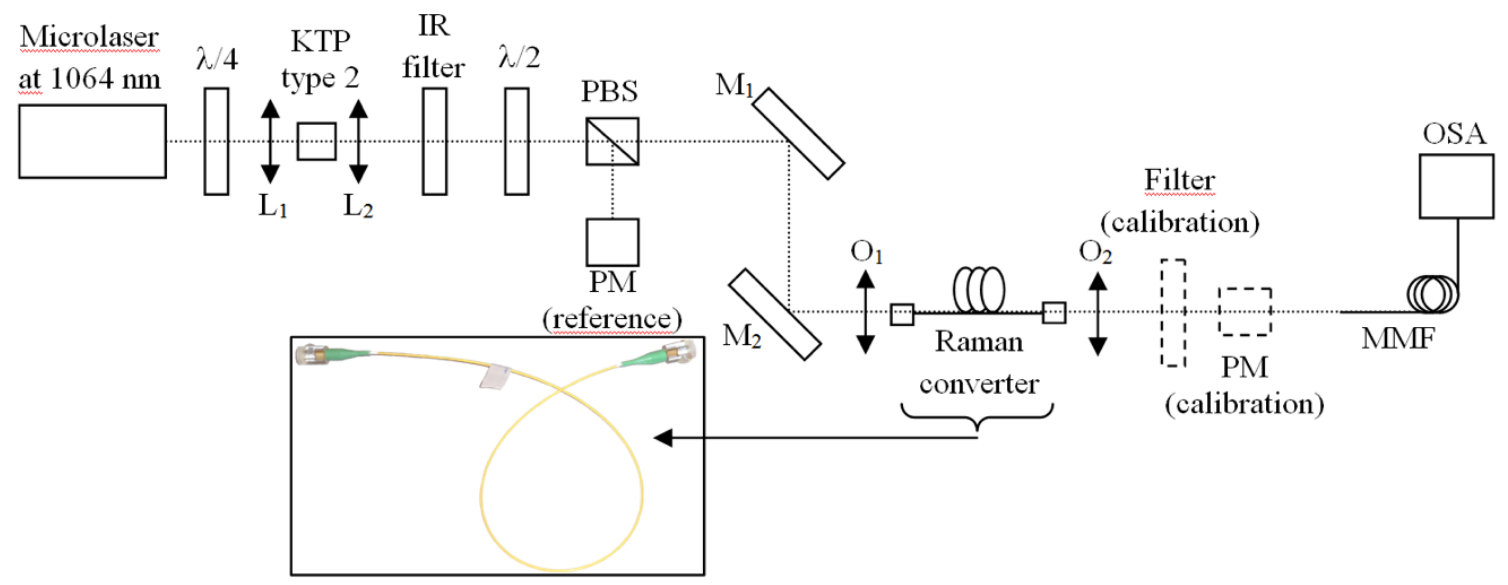

Figure 2. Experimental setup. The $\lambda / 4$ plate enables to optimize the conversion efficiency of the IR beam to the $532 \mathrm{~nm}$ in the type $2 \mathrm{KTP}$ crystal. L1 : focalizing lens. L2 : collimating lens. The $\lambda / 2$ plate associated with the polarizing beam splitter (PBS) enables to vary the incident pump power. The rejected beam is sent to a powermeter (PM reference) to serve as a pump power reference. M1, M2 : mirrors. O1, O2 : microscope objectives. MMF : multimode fiber. OSA : optical spectrum analyzer. In dashed line, removable coloured filter and powermeter (PM calibration) used for the power calibration. Insert : photo of the Raman converter. 


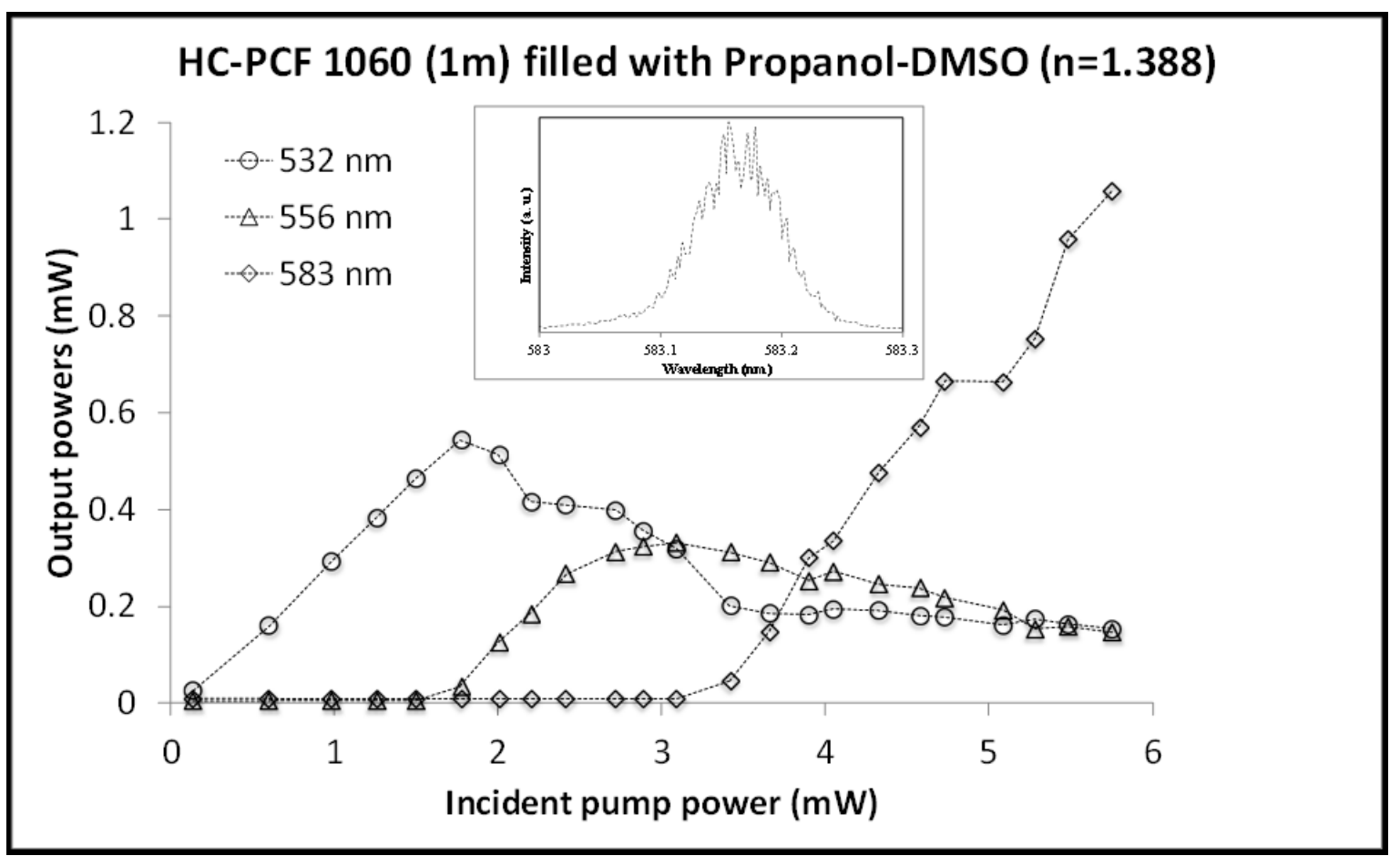

Figure 3. Mean output powers of the different lines emitted by the Raman converter at $583 \mathrm{~nm}$ versus the mean incident pump power. Insert : example of spectrum of the line at $583 \mathrm{~nm}$ at the highest resolution of the OSA.

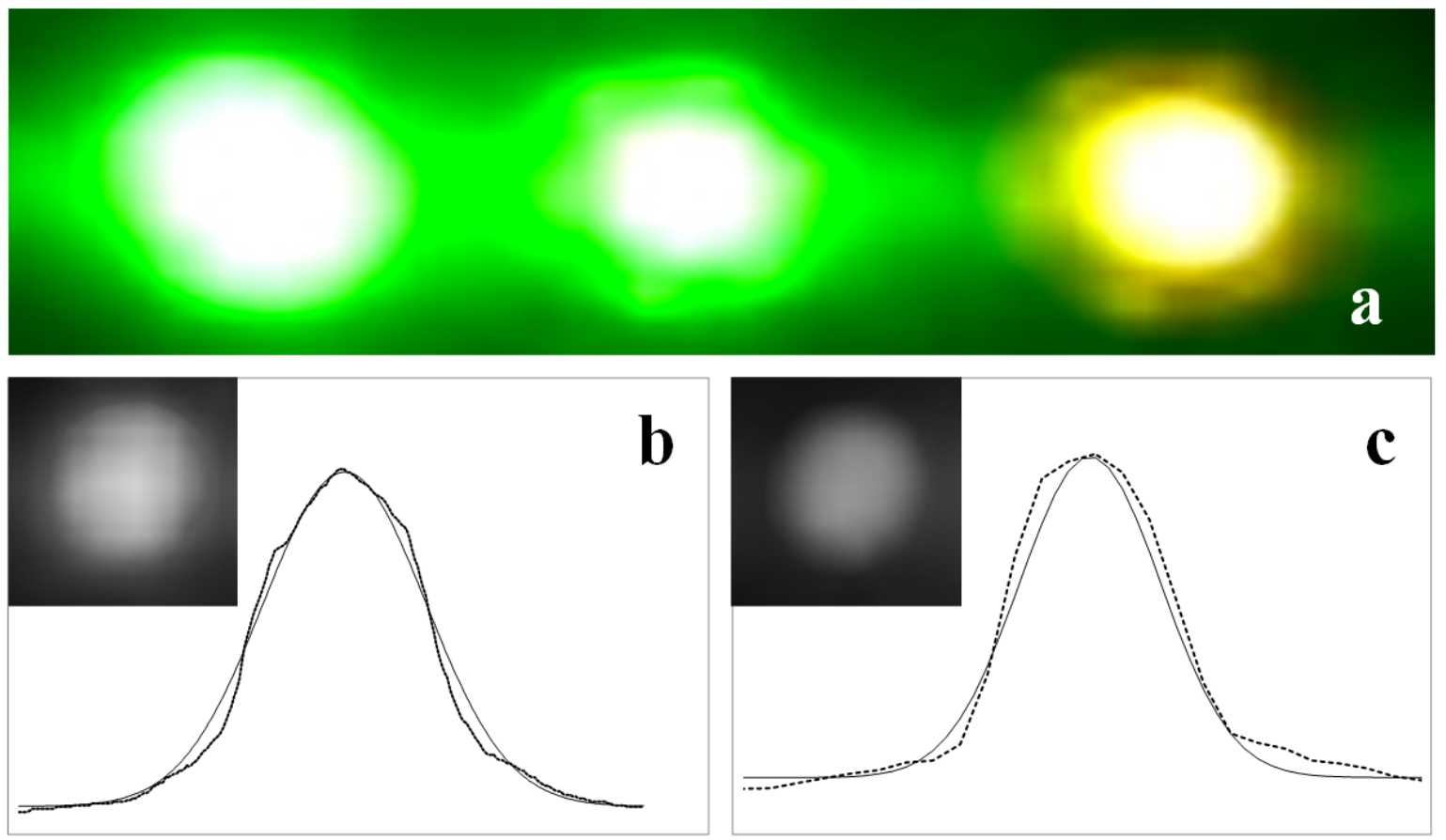

Figure 4. (a) Spatial modes at $532 \mathrm{~nm}, 556 \mathrm{~nm}$ and $583 \mathrm{~nm}$ (from left to right) at the output of the Raman converter around maximum incident pump power. (b) Example of far field pattern of the line at $556 \mathrm{~nm}$ (c) Example of far field pattern of the line at $583 \mathrm{~nm}$ (dotted line : experimental data, full line : Gaussian fit). 